kunft die bisherige lagerübergreifende Kooperation fortsetzen oder ein lagerbildendes Bündnis (im Falle der Christdemokraten ein Mehrheitsbündnis mit den Konservativen und den Liberalen) eingehen wollen. Die Wahl des Parlamentspräsidenten deutete auf eine Fortsetzung der „informellen Großen Koalition“ hin.

\title{
Der Europaausschuss der 16. Wahlperiode: starke Struktur, unambitioniert in eigenen Rechten
}

\author{
René Brosius-Linke*
}

Selten waren Abgeordnete, deren Gesetz(e) vom Bundesverfassungsgericht für verfassungswidrig erklärt wurde(n), so zufrieden mit einer Entscheidung wie bei dem Verfahren um den Vertrag von Lissabon. Kernaussage des Gerichtes ist, dass die eigens geschaffenen Beteiligungsrechte des Gesetzgebers in Angelegenheiten der Europäischen Union den Anforderungen des Grundgesetzes nicht genügen. Das müsste eigentlich verwundern, denn das Thema dominierte die gesamte 16. Wahlperiode des Bundestages. Es wurden neue Beteiligungsformen $^{1}$ vereinbart, die Geschäftsordnung ${ }^{2}$ verändert und schließlich im Zuge der parlamentarischen Ratifizierung des Vertrages von Lissabon ein neues Beteiligungsgesetz ${ }^{3}$ geschaffen.

Insbesondere diejenigen Abgeordneten, die nahe an den Geschehnissen ${ }^{4}$ waren, blickten vor dem Urteil erwartungsfroh nach Karlsruhe. Fraktionsübergreifend wurde mit dem „Einziehen von Leitplanken in Bezug auf die Stellung des Parlaments“ gerechnet. Viel mehr als von der Mehrheit der Abgeordneten erhoffte man sich von den Bundesverfassungsrichtern praxisgerechte Trennlinien zwischen legitimatorischen Anforderungen einerseits und europäischen Zwängen andererseits. Doch warum konnte sich die Mehrheit der Abgeordneten in der Vergangenheit nicht selbst dazu durchringen, diese (gewünschten) Leitplanken einzuziehen? Warum blieb am Ende nur ein hoffnungsvoller Blick nach Karlsruhe? Deutlicher konnte man im Vorfeld der Entscheidung eigenes Scheitern kaum eingestehen. Jedenfalls in Bezug auf die Mitwirkung in Angelegenheiten der EU bestand ${ }^{5}$ mangelnde Bereitschaft oder reale Unfähigkeit des Parlaments, eigene Rechte gegenüber Regierungsvertretern durchzusetzen. Ob der europäische Einfluss auf die deutsche Gesetzgebung bei 80 oder

* Der Autor war von 2005 bis 2009 wissenschaftlicher Mitarbeiter des europapolitischen Sprechers der FDP-Bundestagsfraktion Markus Löning. Der Beitrag gibt ausschließlich seine persönliche Auffassung wieder.

1 Insbesondere die Vereinbarung zwischen der Bundesregierung und dem Bundestag in Angelegenheiten der Europäischen Union (BBV).

2 Änderung GO-BT vom 29. Juli 2008, BGBl. I, S. 1712.

3 BT-Drs. 16/8489.

4 Insbesondere die Obleute des Europaausschusses und Vertreter des Rechtsausschusses.

5 Inwiefern sich dieser Umstand durch die Ende August neu geschaffenen Beteiligungsrechte ändern wird, bleibt einer weitergehenden Untersuchung vorbehalten. 
unter 40 Prozent $^{6}$ angesetzt wird, spielt letztlich keine Rolle. Entscheidend ist, dass der Umsetzungs- beziehungsweise Mitwirkungsspielraum des Bundestages ${ }^{7}$ aufgrund fehlender Beteiligung stetig sank.

Dies müssen die Verfassungsrichter vor Augen gehabt haben, denn im Grunde haben sie dem Gesetzgeber partielle Befassungspflichten auferlegt. Dass vor allem der Bundestag an den erhöhten Mitwirkungsanforderungen des Vertrages von Lissabon (von selbst) erstarke, glaubten die Richter in Karlsruhe offensichtlich nicht. Eine (Teil-)Antwort, ob dieses Misstrauen berechtigt war, gibt der Blick auf die Bemühungen der 16. Wahlperiode, die Beteiligungsrechte des Parlaments auszubauen. Eine eindrücklichere Antwort liegt darin, wie mit diesen Rechten umgegangen worden ist, denn dem Urteil war ein langer Weg parlamentarischer Misserfolge vorausgegangen.

In diesem Kontext ist die Arbeit des Europaausschusses des Bundestages besonders zu betrachten. Als exponierter Akteur in Angelegenheiten der EU ist seine Arbeit mit der des Gesamtparlaments eng verbunden. Seine Niederlagen sind deshalb immer auch die des ganzen Bundestages.

\section{Der Europaausschuss: Starke Struktur, unklare Aufgabe}

Nach den gescheiterten Referenden in Frankreich und den Niederlanden im Frühjahr 2005 verloren sich die institutionellen Reformen der EU in einer ausufernden Denkpause. Die rot-grüne Bundesregierung hatte noch im Oktober 2005 (aber nach der verlorenen Bundestagswahl) den Weg für die Eröffnung der Beitrittsverhandlungen mit der Türkei und Kroatien freigemacht, und die finanzielle Vorausschau 2007 bis 2013 wurde im Spätherbst 2005 verhandelt, also zu einem Zeitpunkt, als der frisch gewählte Bundestag und die Ausschüsse sich gerade konstituierten ${ }^{8}$. Dem gemäß Art. 45 S. 1 GG eingerichteten Ausschuss für die Angelegenheiten der Europäischen Union gingen also die politischen Themen aus. Ohne eigenen Haushalt und ohne eigenes Ministerium fehlen ihm ohnehin die klassischen Arbeitsfelder und natürlichen Gegenspieler in der Bundesregierung.

Geschaffen wurde der Ausschuss, um die dem Bundestag in Art. 23 GG übertragenen Mitwirkungsaufgaben effektiv wahrzunehmen ${ }^{9}$. Diese teilen sich in Informationsrechte gemäß Art. 23 Abs. 2 S. 2 GG und Mitwirkungsrechte gemäß Art. 23 Abs. 3 S. 1 und 2 GG. Beide Aufgaben konnte der Europaausschuss in der 16. Wahlperiode nur unzureichend wahrnehmen. Dies hat vor allem strukturelle Ursachen, denn ein einziger Ausschuss kann schwerlich Informationen für das gesamte Parlament entgegennehmen. Warum sollte zum

6 Vgl. Tilmann Hoppe, Die Europäisierung der Gesetzgebung: Der 80-Prozent-Mythos lebt, in: EuZW 2009, S. 168; Annette Elisabeth Töller, Mythen und Methoden. Zur Messung der Europäisierung der Gesetzgebung des Deutschen Bundestages jenseits des 80-Prozent-Mythos, in: ZParl, 39. Jg. (2008), H. 1, S. 3 - 17, S. 6.

7 Hier sei explizit auf den Bundestag abgestellt. Insbesondere bei der sekundärrechtlichen Gesetzgebung bestehen zu den Ländern Unterschiede.

8 Auch hier ist ein „Webfehler" Europas auszumachen. Im innerstaatlichen Kontext werden Gesetze in Umbruchs- oder Urlaubszeiten nicht beraten. Im europäischen Kontext beraten andere. Am Ende fehlt eine parlamentarisch abgesicherte deutsche Stimme.

9 Vgl. Rupert Scholz, in: Theodor Maunz / Günter Dürig (Hrsg.), Grundgesetz. Kommentar, Art. 45, Rn. 1. 
Beispiel der Finanzausschuss, wenn er Berichte des ECOFIN ${ }^{10}$ von der Bundesregierung erhalten möchte, sich eines Europaausschusses als Mittler bedienen? Dieser besteht weder überwiegend aus Finanzexperten ${ }^{11}$, noch steckt er in der finanzpolitischen Diskussion.

Auch Stellungnahmen nach Art. 23 Abs. 3 S. 1 GG obliegen eindeutig dem Plenum. Ein anderes Ergebnis, etwa ein Selbsteintrittsrecht, lässt sich weder aus Art. 45 S. 2 GG noch, wie vertreten wird, aus der Geschäftsordnung des Bundestages oder den Grundsätzen des Europaausschusses überzeugend entnehmen ${ }^{12}$. Zwar besteht theoretisch die Möglichkeit, den Europaausschuss zu ermächtigen, Stellungnahmen im Namen des Bundestages abzugeben, dies ist aber seit Bestehen dieser Möglichkeit nicht geschehen ${ }^{13}$. Zu sehr ist das Ressortdenken auch unter den Abgeordneten ausgeprägt, als dass man - insbesondere wichtige - Entscheidungen an einen anderen Ausschuss delegieren würde.

Übrig blieb daher im Wesentlichen, sich von der Bundesregierung ihr europäisches Handeln skizzenhaft ${ }^{14}$ erläutern zu lassen - regelmäßig mit wochenlanger Verzögerung und weniger detailliert als ein gut recherchierter Zeitungsbeitrag. Nur wenige politische Themen unterbrachen dieses bleierne Nachvollziehen von exekutiven Schritten ${ }^{15}$, darunter die Beitrittsdiskussion um Bulgarien und Rumänien, die Einrichtung der EU-Grundrechteagentur, Mehrsprachigkeit in der EU und vor allem der Vertrag von Lissabon, einschließlich der damit zusammenhängenden institutionellen Anpassungen ${ }^{16}$. Während die Fragen der Erweiterung und Mehrsprachigkeit hoch emotional debattiert wurden, konnte der Europaausschuss seine institutionell gute Ausgangslage in Bezug auf die Ausgestaltung parlamentarischer Rechte nicht nutzen.

\section{Anpassungen der Beteiligungsrechte als (unlösbare) Daueraufgabe?}

Aufgrund des Mangels an politischen Themen kommt der Arbeit des Europaausschusses an den Beteiligungsrechten, insbesondere mit Blick auf das darauffolgende Lissabon-Urteil am Ende der Wahlperiode, eine besondere Bedeutung zu. Denn das BVerfG hatte sich gerade mit der Frage zu beschäftigen, ob die vorhandenen innerstaatlichen Institutionen sowie deren Anpassungsfähigkeit an europäische Entscheidungsformen im Zweifelsfall ausreichen würden, um der theoretisch möglichen (missbräuchlichen) Anwendung von primärrechtlichen (unbestimmten) Rechtsgrundlagen entgegenzuwirken.

An Analysefähigkeit fehlte es in der 16. Wahlperiode nicht, denn die Missstände als solche wurden, wie sich aus zahlreichen Parlamentsdebatten und Protokollen ergibt, im ausrei-

10 Die Ratsformation auf Ebene der Finanzminister.

11 Hans Eichel war in der 16. Wahlperiode eine Ausnahme.

12 Jede Auslegung der GO-BT scheitert letztlich am Wortlaut von Art. 45 GG.

13 Vgl. Rupert Scholz, a.a.O. (Fn. 9), Beck-Onlinekommentar, Art. 45, Rn. 2. Trotz dieses Umstandes wurde genau dieser Bereich aktuell weiter gestärkt und ein rein theoretisches Verfassungsrecht geschaffen.

14 Was in durchschnittlich drei Sitzungen pro Monat jeweils ein bis zwei Stunden pro Sitzung geschah. Kleinere Berichterstattergespräche gab es nur selten.

15 Der Europaausschuss spielte zum Beispiel keine aktive Rolle bei der deutschen EU-Ratspräsidentschaft im ersten Halbjahr 2007 oder bei EU-Gipfeln. Seine Aufgabe beschränkte sich klar auf das Entgegennehmen von Berichten, teils auf Beamtenebene vorgetragen.

16 Die Beratungen um das Begleitgesetz II im August und September 2009 in Folge des Urteils werden hier nicht berücksichtigt. 
chenden Maß identifiziert. Auch gab es genügend Gelegenheiten, dieser misslichen Mechanik entgegenzuwirken. Zum einen hätte dies bei den Verhandlungen um die Vereinbarung zwischen Bundesregierung und Bundestag in den Angelegenheiten der Europäischen Union $\left(\mathrm{BBV}^{17}\right)$ geschehen können, zum anderen bot sich eine große Chance im Zuge der Ratifizierung des Vertrages von Lissabon. Wie immer bei Primärrechtsänderungen ist der Moment der Hoheitsübertragung geeignet, Mitwirkungsrechte neu und parlamentsfreundlicher zu gestalten, denn Art. 23 Abs. 1 S. 3 GG gebietet hier regelmäßig eine verfassungsändernde Mehrheit ${ }^{18}$. Insbesondere der Vertrag von Lissabon eignete sich dazu hervorragend. Die Akteure auf exekutiver Seite, insbesondere die deutsche Ratspräsidentschaft im Frühjahr 2007, standen unter enormem Erfolgsdruck, während die nationalen Parlamente die Brückenfunktionen zur Bevölkerung bieten konnten, die der Exekutive offenbar verloren gegangen war, wie die Referenden in Frankreich und den Niederlanden gezeigt hatten.

In beiden Fällen ist es dem Bundestag nicht gelungen, substantielle Verbesserungen herzustellen. Die BBV kann zwar inhaltlich als Fortschritt bezeichnet werden ${ }^{19}$, jedoch verzichtete man bis zum Verfassungsgerichtsurteil auf eine gesetzliche Ausgestaltung der damit formulierten Rechte und beließ es bei einem verfassungsrechtlichen Vertrag. Die Folge war, dass das Parlament im weiteren Verlauf bei Auslegungsfragen stets unterlag. Gänzlich versagt hat das Parlament bei den Beratungen um das Begleitgesetz I.

\section{Oktober 2005 bis September 2006: zweiter Frühling für das Parlament}

Erst ein Jahrzehnt nach der Maastricht-Entscheidung des BVerfG ${ }^{20}$ wurde im Bundestag deren Bedeutung für die tägliche Arbeit (neu) erkannt und akzeptiert. Den Befürwortern einer weitgehenden europäischen Integration war es lange Zeit gelungen, die strengen Maastricht-Auflagen der Verfassungsrichter, die überwiegend demokratietheoretischer Natur waren, als Euroskepsis zu deuten. Staatstheoretische Bedenken sollten keinen Einfluss auf die integrationspolitische Willensbildung haben. Diese Grundstimmung veränderte sich erst mit dem Verfassungskonvent 2002/2003 und der Diskussion um die Ratifizierung der europäischen Verfassung in Deutschland. Die Beteiligung des Bundestages im (europäischen) Rechtsetzungsgefüge spielte nunmehr eine gewichtigere Rolle - allerdings mit anderen Vorzeichen als in der darauffolgenden Wahlperiode. CDU/CSU sowie FDP waren darum bemüht, die rot-grüne Bundesregierung, die mit hauchdünner wie fragiler Mehrheit regierte, auf allen Feldern zu stellen. In dieser Konstellation hatten die Parlamentsrechte objektiv gesehen gute Karten. Dies zeigte sich etwa im Zuge der Ratifizierung des Verfassungsvertrages im Mai 2005, als eine entsprechende Änderung des EUZBB ${ }^{21}$ beschlossen wurde.

17 BT-Drs. 16/2620.

18 Vgl. Rupert Scholz, a.a.O. (Fn. 9), Beck-Onlinekommentar, Art. 23, Rn. 21 mit weiteren Nachweisen; dabei zielte die Kernfrage der Entscheidung darauf ab, wo die Grenzen einer Hoheitsübertragung festzulegen sind.

19 Diese soll trotz des neuen Zusammenarbeitsgesetzes erhalten bleiben und gegebenenfalls in der 17. Wahlperiode erneut ausgebaut werden.

20 BVerfGE 89, S. $155 \mathrm{ff}$.

21 Gesetz vom 12. März 1993, BGBl. I, S. 311, S. 1780; zuletzt geändert durch Art. 2 Abs. 1, Gesetz vom 17. November 2005, BGBl. I, S. 3178; Geltung ab 1. November 1993. 
Das Scheitern der Referenden in Frankreich und den Niederlanden nur einen Monat später und die vorgezogene Bundestagswahl im September 2005 änderten die Rahmenbedingungen erheblich. Die Abgeordneten der 16. Wahlperiode wollten nicht mehr auf die Ratifizierung (irgend-)einer EU-Reform warten. Grund dafür gab zusätzlich die öffentliche Diskussion um die Entstehungsgeschichte der Antidiskriminierungsrichtlinie, die sich sogar als Wahlkampfthema zu eignen schien. Ebenso wollte man den Eindruck korrigieren, den die Parlamentarier in der mündlichen Verhandlung des Bundesverfassungsgerichts zum Europäischen Haftbefehl $^{22}$ hinterlassen hatten, darunter die Bemerkung des damaligen Vertreters der grünen Regierungsfraktion Christian Ströbele, der äußerte, er habe sich „normativ unfrei gefühlt“.

Maßgeblich beteiligt an diesem Bestreben war der ehemalige europapolitische Sprecher der Unionsfraktion Peter Hinze, der in der neu gebildeten Bundesregierung Europastaatssekretär geworden war. Auf dessen Betreiben wurde das „vorzeitige“ Umsetzen von $\$ 6$ des Gesetzes über die Zusammenarbeit von Bundestag und Bundesregierung in Angelegenheiten der Europäischen Union (EUZBBG) in den Koalitionsvertrag 23 geschrieben. Nach kurzer Zeit begannen alle Fraktionen, die Zusammenarbeit von Bundestag und Bundesregierung neu zu verhandeln. Unter Federführung des Auswärtigen Amtes wurde über einen Zeitraum von wenigen Monaten ein Vereinbarungstext zusammengestellt und abgestimmt, der sich an der bereits 1993 geschlossenen und 1998 ${ }^{24}$ überarbeiteten Vereinbarung zwischen der Bundesregierung und den Ländern ${ }^{25}$ sowie an einen Gesetzentwurf der CDU/ CSU-Fraktion der 15. Wahlperiode ${ }^{26}$ orientierte. Dem Bundestag sicherte die BBV eine Vielzahl von Informationen zu sowie in speziell geregelten Fällen mündliche Unterrichtungen und Mitwirkungsrechte ${ }^{27}$. Neu war vor allem eine detaillierte Aufzählung, welche Vorhaben die Bundesregierung dem Parlament zuleiten muss und für welche sie spezielle Berichtsbögen erstellen sollte ${ }^{28}$.

Bereits bei den Verhandlungen um die BBV fand eine Diskussion darüber statt, ob die Ziffer VI „Beitritt und Vertragsrevision“ in dieser Form in der Vereinbarung enthalten sein sollte beziehungsweise konnte. Diesen Passus hatte die CDU/CSU-Fraktion in den genannten Gesetzentwurf hineingeschrieben, da zu diesem Zeitpunkt die Beitrittsverhandlungen mit der Türkei und Kroatien noch nicht aufgenommen waren und man sich in Oppositionszeiten des Parlamentsvorbehalts in Beitrittsfragen ${ }^{29}$ sicher sein wollte. Nach der förmlichen Aufnahme der Beitrittsverhandlungen im Herbst 2005 war der Union dieser Passus weniger wichtig. Aus Gründen der Stärkung der Parlamentsrechte blieb er jedoch in der BBV enthalten, und gerade hieran zeigte sich später in der Wahlperiode die fehlende Durchsetzungsfähigkeit des Parlaments gegenüber der Regierung.

22 BVerfGE 113, S. 273 ff.

23 Koalitionsvertrag von CDU, CSU und SPD vom 11. November 2005, S. 148, http://www.cducsu.de/upload/koavertrag0509.pdf (Abruf am 29. November 2009).

24 Welche ihrerseits im Jahr 2008 erneut umfassend überarbeitet wurde.

25 Bund-Länder-Vereinbarung vom 29. Oktober 1993, in: Bundesanzeiger, Nr. 226 vom 2. Dezember 1993, S. 10425; ergänzt durch Vereinbarung vom 8. Juni 1998, in: Bundesanzeiger, Nr. 123 vom 8. Juli 1998, S. 9433.

26 BT-Drs. 15/4716.

27 Eingehend dazu Tilmann Hoppe, Drum prüfe, wer sich niemals bindet. Die Vereinbarung zwischen Bundesregierung und Bundestag in Angelegenheiten der Europäischen Union, in: DVBl. 2007, S. $1540 \mathrm{ff}$.

28 Anlage 1 und 2, BT-Drs. 16/2620.

29 Insbesondere wohl im Fall der Türkei. 
Im September 2006 wurde die Vereinbarung zwischen der Bundesregierung und dem Bundestag offiziell von Vertretern des Bundestages sowie der Bundeskanzlerin unterzeichnet, vom Bundestag angenommen ${ }^{30}$ und im Bundesanzeiger veröffentlicht ${ }^{31}$. Damit war das erste Teilziel erreicht. Die 16. Wahlperiode schien die Lücke zwischen dem eigens im Rahmen der Ratifizierung des Vertrages von Maastricht eingesetzten Sonderausschuss „Europäische Union"32 und der Gegenwart überbrückt zu haben.

\section{BBV im Praxistest}

Die Umsetzung der BBV erwies sich als das härtere Terrain, wie die Diskussion um die EUGrundrechteagentur als Rechtsetzungsakt im Sinne von Art. 23 Abs. 3 S. 2 GG und das Verhandlungsmandat zum Vertrag von Lissabon zeigen. Die Beispiele eignen sich besonders, denn sie standen zu einer Zeit auf der Tagesordnung, als sich der Bundestag mit der BBV gerade erst eine neue Grundlage der Zusammenarbeit mit der Bundesregierung gegeben hatte, die er als ausreichend empfand.

\subsection{Die „Mitwirkung“ im Fall der Grundrechteagentur}

An der Debatte um die Europäische Grundrechteagentur im zweiten Halbjahr 2006 bis März 2007 lassen sich nahezu sämtliche europapolitischen Kritikpunkte - Subsidiarität, fehlende Rechtsgrundlage, überbordende Bürokratie, hohe Kosten, Konkurrenz zu bestehenden Strukturen - illustrieren ${ }^{33}$. Außerdem basiert die Agentur auf einer EU-Verordnung, also einem Instrument des übertragenen Rechts.

Bereits im Dezember 2003 legte der Europäische Rat den Grundstein für eine ganze Reihe von Gemeinschaftsagenturen, darunter die Agentur für Flugsicherung in Köln, die Europäische Eisenbahnagentur in Lille-Valenciennes oder die Europäische Agentur für Seesicherheit mit Sitz in Lissabon ${ }^{34}$. Darüber hinaus einigten sich die Staats- und Regierungschefs informell auch auf die Schaffung weiterer Agenturen in der Zukunft. Der regionalen Erweiterung der Union im Jahr 2004 folgte eine zahlenmäßige „Anpassung“: Waren es im Jahr 2000 gerade mal acht Agenturen, wurden mittlerweile 27 (Gemeinschafts-)Agenturen in 27 Mitgliedstaaten geschaffen ${ }^{35}$. Die jährlichen Gesamtkosten belaufen sich auf circa 1,5

30 BT-Plenarprotokoll 16/52 vom 22. September 2006, S. 5053A - 5068C.

31 BT-Drs. 16/2620; auf dieses Verfahren einigte man sich, um rechtsverbindlich genug zu sein, ohne jedoch die Gesetzesbindung zu haben.

32 Beim so genannten Maastricht-Ausschuss handelte es sich um einen Sonderausschuss für den Ratifizierungsprozess des Vertrages über die Europäische Union (Maastricht-Vertrag). Er hatte die Aufgabe, die Vertragsverhandlungen zu begleiten und die damit verbundenen Grundgesetzänderungen vorzubereiten. Dieser aus 39 Abgeordneten zusammengesetzte Ausschuss bestand trotz des seit 1991 etablierten EG-Ausschusses.

33 Dies spiegelt sich letztlich im Urteil zu Art. 352 AEUV wider.

34 Vgl. Website des Rates, http://www.consilium.europa.eu/ueDocs/cms_Data/docs/pressData/de/ ec/79654.pdf (Abruf am 27. November 2009).

35 Eine vollständige Liste ist auf der Website der Europäischen Union zu finden, http://europa.eu/ agencies/index_de.htm (Abruf am 27. November 2009). 
Milliarden Euro ${ }^{36}$. In diesem Zusammenhang kam es im Zuge einer Paketlösung auch zur Zusage, dass die 1998 eingerichtete Europäische Stelle zur Beobachtung von Rassismus und Fremdenfeindlichkeit ${ }^{37}$ in Wien zur Europäischen Agentur hochgestuft werden soll$\mathrm{te}^{38}$. Ohne dieses Zugeständnis wäre ein Beschluss über die anderen Agenturen durch die nicht bedachten Mitgliedstaaten blockiert worden.

Die Umsetzung des Beschlusses des Europäischen Rates erfolgte in einer denkbar ungünstigen Zeit. Am 30. Juni 2005, also keine vier Wochen nach dem Scheitern der Referenden über die Europäische Verfassung in Frankreich und den Niederlanden ${ }^{39}$, legte die Kommission einen entsprechenden Verordnungsentwurf vor, der die Gründung einer EUGrundrechteagentur vorsah ${ }^{40}$. Der Zweck der Agentur war von Beginn an unklar. Sie soll Werbung für die EU-Grundrechtecharta machen, EU-Institutionen bei der Implementierung von Grundrechten beraten, über europäische Grundrechte aufklären, Studien über die unterschiedliche strafrechtliche Verfolgung von fremdenfeindlichen Übergriffen erstellen ${ }^{41}$ und die Mitgliedstaaten auf freiwilliger Basis bei der Implementierung europäischer Grundrechte in die innerstaatliche Ordnung unterstützen ${ }^{42}$.

Doch gab es zu diesem Zeitpunkt keine ureigenen EU-Grundrechte ${ }^{43}$. Kritiker hielten die Agentur folglich für überflüssig ${ }^{44}$, da sie zum Gründungszeitpunkt ohne Verfassungsvertrag in Bereichen tätig werden solle, die grundsätzlich in innerstaatlicher Regie durch die Rechtsabteilungen und die Öffentlichkeitsarbeit der Ministerien durchgeführt werden konnten. Entsprechend des unklaren Aufgabenprofils der Agentur, fand sich keine andere Rechtsgrundlage als Art. 308 EGV $^{45}$, der als Tatbestandsmerkmal beinhaltet - daran sei an dieser Stelle nochmals erinnert -, dass die Gemeinschaft tätig werden könne, um im „Rahmen des Gemeinsamen Marktes (!) eines ihrer Ziele zu verwirklichen“. Zwar sieht Art. 308 EGV weder in der aktuellen noch in der überarbeiteten Fassung des Vertrages von Lissabon (Art. 352 AEUV) eine direkte Beteiligung der nationalen Parlamente vor; allerdings räumt das Einstimmigkeitserfordernis der Norm in Verbindung mit dem Mitwirkungsregime des Grundgesetzes dem Bundestag ein weitgehendes politisches Vetorecht ein ${ }^{46}$.

36 Vgl. Website des Europäischen Parlements, http://www.europarl.europa.eu/oeil/FindByProcnum. do?lang=en\&procnum=INI/2008/2207 (Abruf am 27. November 2009).

37 Sicherlich hatte auch das Erstarken der FPÖ Ende der 1990er Jahre einschließlich der Regierungsbeteiligung ab 1999 etwas damit zu tun.

38 Dieses Verfahren der Gründung eines Koordinierungsbüros, das später eine Agentur wird, ist aktuell beim Asylunterstützungsbüro zu beobachten.

39 Ebenfalls mitten in der Sommerpause aller nationalen Parlamente und im Bundestagswahlkampf in Deutschland.

40 Vgl. Website des Rates, http://ec.europa.eu/justice_home/doc_centre/rights/doc/com_2005_280_ de.pdf (Abruf am 27. November 2009).

41 Allerdings gemäß Art. 4 Abs. 1a des Verordnungsvorschlages nur auf Basis von (freiwillig) durch die Mitgliedstaaten zur Verfügung gestellten Daten.

42 Umfassend dazu Ines Härtel, Die Europäische Grundrechteagentur: unnötige Bürokratie oder gesteigerter Grundrechtsschutz?, in: EuR 2008, S. 489 ff.

43 Mit Ausnahme der mitgliedstaatlichen Verweise in Art. 6 II EGV.

44 BT-Plenarprotokoll 16/79 vom 1. Februar 2007, S. 7921B - 7922A, S. 7944A.

$45 \mathrm{Im}$ Verordnungsvorschlag dagegen sollte die Agentur auf Art. 30, 31 und 34 Abs. 2 Buchstabe c gestützt werden.

46 Inwieweit die im Jahr 2003 gegebene informelle Zusage auf Regierungsebene deshalb überhaupt mit Art. 23 Abs. 2 S. 2 und Abs. 3 S. 1 GG vereinbar war - schließlich handelte es sich um einen Rechtsakt in diesem Sinne -, ist einer anderen Untersuchung vorbehalten. Die Verlagerung von 
Im Bundestag kam es im Jahr 2006 vor allem auf Initiative der $\operatorname{FDP}^{47}$ zu kritischen Fragen. Neben grundsätzlicher Kritik an solchen Agenturen bezüglich fehlender Zuordnungsmöglichkeit im staatsrechtlichen Gefüge, fehlender demokratischer Legitimation und Rechtsschutz sowie finanzieller Konkurrenz zum Europäischen Gerichtshof für Menschenrechte ${ }^{48}$ war der Ausgangspunkt des Streites, dass nach den gescheiterten Referenden über die Europäische Verfassung die EU-Grundrechtecharta keine Rechtsverbindlichkeit besaß. Es gab keinen rechtlichen $\mathrm{Maßstab}^{49}$, an dem sich eine „Grundrechteagentur" verbindlich orientieren konnte. Ihre Einrichtung erschien also zu diesem Zeitpunkt - die EU befand sich in einer „Denkpause" 50 - neben der grundsätzlichen Kritik unsinnig, kostete Millionen Steuergelder und ignorierte auf eindrucksvolle Weise den Bürgerwillen in Frankreich und den Niederlanden. Ein weiterer Punkt war die im Raum stehende Frage, inwieweit sich ein einmal in Gang gesetztes Verfahren der europäischen Rechtsetzung überhaupt aufhalten beziehungsweise beeinflussen lassen würde ${ }^{51}$.

Mit der Forderung nach einer Aussetzung des Gründungsvorgangs war die FDP nicht allein. Die Fraktion der Grünen ${ }^{52}$ und viele Abgeordnete der Regierungsfraktionen ${ }^{53}$ teilten diese Bedenken und forderten den (vorläufigen) Stopp der Agentur bis zur Rechtsverbindlichkeit der EU-Grundrechtecharta. Die Bundesregierung gebrauchte die schwache Position der Agenturbefürworter auf europäischer Ebene dazu, ihrerseits die Gründung als Druckmittel in anderen Verhandlungen zu benutzen. Als im Frühsommer 2006 der Druck auch innerhalb der Regierungsfraktionen zunahm, sahen die Abgeordneten die Gefahr, dass die Bundesregierung auf dem Gipfel Mitte Juni 2006 der Gründung zustimmen werde, um nicht ihrerseits in eine Situation zu geraten, die bereits gegebene grundsätzliche Zustimmung zur Agentur im Jahr 2003 wegen eines Parlamentsvorbehalts revidieren zu müssen ${ }^{54}$. Um dem vorzubeugen, entschieden die Obleute des federführenden Europaausschusses zunächst, dass man aus Effektivitäts- und Zeitgründen von einer Stellungnahme nach Art. 23 Abs. 3 S. 1 GG absehen und mittels eines gemeinsamen Briefs der Obleute der Fraktionen an die Bundeskanzlerin und den Außenminister herantreten werde ${ }^{55}$. In dem Schreiben vom 8. Juni 2006 baten die Obleute im Namen aller Fraktionen, der Gründung der Agentur auf dem Gipfel vom 15./16. Juni 2006 nicht zuzustimmen. Gleichzeitig wurde darauf

materiellem Recht (Rahmenbeschlüsse) in die erste Säule durch den Vertrag von Lissabon reduziert auf ein absolutes Mindestmaß. Auch hier haben die nationalen Parlamente durch den Lissabon-Vertrag unkompensiert an Einfluss verloren.

47 BT-Drs. 16/3621 vom 29. November 2006.

48 Der Etat der Grundrechteagentur beträgt mittlerweile circa 10 Prozent des gesamten Europarates. Aus dem Etat des Europarates wird der Europäische Gerichtshof für Menschenrechte finanziert.

49 Art. 3 Ziffer 2 des Verordnungsentwurfs bezog sich ausdrücklich auf die EU-Grundrechtecharta. Art. 3 Ziffer 2 der letztlich verabschiedeten Verordnung verzichtet auf einen solchen Bezug.

50 Erstmals fiel der Begriff im Zusammenhang mit Tony Blairs Absage des britischen Referendums am 6. Juni 2005.

51 Im Ergebnis nahm die Agentur sowohl zeitlich als auch finanziell ausgestattet die Arbeit genau so auf, wie es der Verordnungsentwurf vom 30. Juni 2005 vorsah.

52 BT-Drs. 16/3617.

53 Vgl. BT-Plenarprotokoll 16/79, S. 7943B.

54 Ein parlamentarisches „Zurückpfeifen“ gilt als absolut peinlich.

55 Auch hieran zeigt sich eine Schwäche des Art. 23 Abs. 3 S. 1 GG. Über eine Stellungnahme entscheidet das Gesamtplenum in gegebenenfalls zwei Lesungen. Im Regelfall dauert dies mehrere Wochen. 
hingewiesen, dass man aufgrund der zuvor zugesagten restriktiven Position der Bundesregierung vorerst auf einen förmlichen Beschluss nach Art. 23 Abs. 3 GG verzichte.

Das Schreiben führte zu einem unerwarteten Ergebnis: Zwar wurde die Agentur nicht im Juni 2006 formal beschlossen, sondern am 4./5. Dezember 2006 einigte sich der Rat für Justiz und Inneres auf exekutiver Ebene ohne Einbindung oder vorherige Information des Bundestages. Während sich die beteiligten Abgeordneten, insbesondere der damalige christlich-demokratische Vorsitzende des Europaausschusses Matthias Wissmann, sicher waren, dass der noch ausstehende formale Gründungsakt im Rat durch die Vertreter Deutschlands bis auf Weiteres auf Eis gelegt sei, wurde man Anfang Dezember 2006 mit der Nachricht überrascht, dass die umstrittene Agentur bereits zum 1. Januar 2007 - also zu Beginn der deutschen Ratspräsidentschaft - ihre Arbeit aufnehmen würde ${ }^{56}$. Die Bundesregierung hatte, ohne vorherige Ankündigung an das Parlament, im Ministerrat mittels einer politischen Einigung (A-Punkt) zur Gründung der Agentur grünes Licht gegeben, um die Diskussion nicht in ihrer Präsidentschaft führen zu müssen. Zu weiterem Unmut führte es, dass die Agentur ohne förmlichen Ratsbeschluss (also ohne Rechtsgrundlage) ihre Tätigkeit begann, denn 2006 gab es keine weitere Ratssitzung mehr. Dieser wurde erst am 15. Februar 2007 auf dem Justiz- und Innenministerrat nachgeholt. Die österreichische Regierung hatte zum Abschluss ihrer Präsidentschaft ihre Agentur in Wien; Deutschland, das seine förmliche Zustimmung über Jahre verweigert hatte, akzeptierte die Arbeitsaufnahme ohne förmlichen Ratsbeschluss.

In der darauffolgenden Sitzung des Europaausschusses am 13. Dezember 2006 versuchten die Vertreter der Bundesregierung, den Eindruck zu erwecken, sie hätten das Anliegen des Bundestages beherzt vertreten und sich als Äquivalent zur Schaffung der Agentur jedenfalls um deren finanzielle Verkleinerung bemüht. Die Abgeordneten hielten dem einen Drahtbericht ${ }^{57}$ der ständigen Vertretung in Brüssel entgegen ${ }^{58}$, demnach kein Ressortminister bei der entscheidenden Abstimmung vor Ort gewesen sei ${ }^{59}$ und der ständige Vertreter im Rat keine Weisung hatte, die Bedenken des deutschen Parlaments vorzutragen. Besonders brisant war die Information, dass die Bundesrepublik mit ihrer Kritik an der Agentur nicht allein gewesen wäre, es also eine echte Chance auf Verhinderung gegeben hätte ${ }^{60}$. Auch Länder wie die Niederlande und Italien teilten nämlich die Kritik.

Die Bedenken des Bundestages jedenfalls blieben in der entscheidenden Ratssitzung im Dezember 2006 unerwähnt, die Intervention des deutschen Parlaments blieb im europäischen Prozess spurenlos. Nicht einmal eine zeitliche Verzögerung konnte erreicht werden, denn bereits der Verordnungsentwurf vom 30. Juni 2005 sah die Arbeitsaufnahme der Agentur zum 1. Januar 2007 vor.

Zum Anwendungsfall der BBV wurde die Agentur, weil die politische Einigung ohne vorherige Information an den Bundestag erfolgte. Entgegen der Zusage gemäß Ziffer I. Abs. 1 der BBV, den Bundestag „frühzeitig und fortlaufend“ über Vorhaben im Rahmen

56 Wie im Verordnungsentwurf vom 30. Juni 2005 anvisiert.

57 Diese wurden gemäß der BBV seit September 2006, jedenfalls auf Verlangen, dem Parlament zugeleitet.

58 Lange war unklar, ob diese Berichte von der BBV erfasst sind.

59 Gemäß der Geschäftsordnung des Rates kann ein einstimmiger Beschluss auch bei Enthaltung und fehlender Anwesenheit getroffen werden.

60 Art. 308 EGV schreibt die Einstimmigkeit im Rat fest. 
der EU zu unterrichten, entgegen der Zusage der Bundesregierung in Ziffer I Abs. 1 UA 4 der BBV, den Bundestag ,über aktuelle politische Entwicklungen im Rahmen der Europäischen Union, auch im Wege der politischen Frühwarnung “ zu informieren, entgegen ihrer Zusage gemäß Ziffer I. Abs. 2c), Berichte der Ständigen Vertretung über Ratssitzungen zu übermitteln, und im Grunde auch entgegen Ziffer II. Abs. 4 der BBV, im Falle einer Stellungnahme des Plenums gemäß Art. 23 Abs. 3 S. 1 GG einen Parlamentsvorbehalt geltend zu machen, wenn wesentliche Belange des Bundestages nicht durchzusetzen sind und vor der abschließenden Entscheidung im Rat Einvernehmen mit dem Bundestag herzustellen, hatte die Bundesregierung grünes Licht für die Gründung der Agentur gegeben.

Das Wissen um die Kritik im eigenen Hause führte so gesehen zu einer Beschleunigung des Vorgangs. Um den formalen Kriterien Rechnung zu tragen, und einer Diskussion um das Einvernehmen zu entgehen, wurde der förmliche Beschluss auf eine Zeit nach der politischen Einigung gelegt. Dieses Verfahren sollte sich im weiteren Verlauf der Wahlperiode aus Sicht der Bundesregierung als erfolgreiche Praxis bewähren.

Die Entscheidung über die Grundrechteagentur zeigt, dass Einflussnahme auf europäische Rechtsetzungsvorhaben, seien es wie in diesem Fall auch nur institutionelle Angelegenheiten, mit erheblichem Aufwand betrieben werden muss. Obwohl dies hier seitens des Bundestages geschehen war, wurde ein Erfolg nicht sichtbar. Entschieden hatten andere Akteure. Leider wurden die (schlechten) Erfahrungen nicht dazu genutzt, sich künftig frühzeitiger in die europäische Rechtsetzungsdebatte einzubringen beziehungsweise die BBV textlich zu ändern. Der Bundestag, der sich mit der BBV an der vordersten Front einer europäischen Bewegung ${ }^{61}$ glaubte, hatte in der praktischen Umsetzung eine herbe Niederlage erlitten. Im weiteren Verlauf der Wahlperiode verzichtete er sogar auf die Forderung, als Zeitpunkt des Einvernehmens einen Termin vor der politischen Einigung im Rat festzulegen.

Im Nachgang der Entscheidung wurden in der Bundesregierung erste Stimmen laut, Drahtberichte, die wie gesehen geeignet waren, die Bundesregierung ernstlich in Bedrängnis zu bringen, nicht weiter an den Bundestag zuzuleiten. Insbesondere unter dem Deckmantel der Vertraulichkeit sollte künftig auf eine förmliche Übermittlung verzichtet werden. Der erste Konflikt über die Auslegung des BBV-Textes war geboren und führte noch zu parlamentarischen Gegenreaktionen ${ }^{62}$. Auf einen Schlag wurde vielen Parlamentariern klar, dass im Konfliktfall die BBV zwar Rechte zusicherte, diese jedoch nicht ohne Weiteres gelten würden. Dennoch einigte man sich später auf einen restriktiveren Umgang mit den Drahtberichten.

\subsection{Das Junktim von Vertragsrevision und Ausbau von Parlamentsrechten}

Nach den Erfahrungen mit der Grundrechteagentur wurden insbesondere die verwaltungstechnischen Voraussetzungen für die Umsetzung der BBV geschaffen. Im Februar 2007 eröffnete die Vertretung des Bundestages in Brüssel. Es wurde das Referat PA1 eingerichtet, das mit der politischen Priorisierung von europäischen Vorhaben betraut wurde, und man

61 Zum Beispiel Forderungen aus den Niederlanden nach einer roten Karte für die nationalen Parlamente.

62 BT-Drs. 16/7139, unter II. Ziffer 6. 
suchte verstärkt nach neuen Informationskanälen durch die Bundesregierung ${ }^{63}$. Trotz der großen politischen Einigkeit während der deutschen Ratspräsidentschaft im ersten Halbjahr 2007, zeigten sich in der Mitte der Wahlperiode die entgegengesetzten Interessen von Legislative und Exekutive häufiger und heftiger.

Mit der Auseinandersetzung um die Mitwirkung des Bundestages an der ordentlichen Vertragsänderung selbst bahnte sich eine weitere Niederlage an. Traditionell war das Verfahren so gestaltet, dass die Bundesregierung gemäß Art. 59 Abs. 2 GG auf Regierungsebene die Vertragsänderungen aushandelte und der Bundestag diese dann gemäß Art. 23 Abs. 1 S. 3 in Verbindung mit Art. 79 Abs. 3 GG ratifizierte.

Um den Forderungen nach einem erneuten Konvent etwas entgegenzusetzen, ging die deutsche Ratspräsidentschaft im Frühsommer 2007 einen anderen Weg. Sie hatte sich im Vorfeld des am 21. Juni 2007 beginnenden EU-Gipfels mit ihren europäischen Partnern auf ein weitestgehend ausformuliertes Verhandlungsmandat ${ }^{64}$ geeinigt, das der Gipfel auf Ebene der Staats- und Regierungschefs nach Widerständen aus Polen letztlich billigte. Gleichzeitig wurde beschlossen, eine Regierungskonferenz durchzuführen, um aus dem Kompromiss einen abstimmungsfähigen Text herzustellen. Ein derart weitreichendes Mandat vor einer Regierungskonferenz war neu und zeigte die gewachsene Bedeutung des Europäischen Rates ${ }^{65}$. Es bot zugleich die Chance für den Bundestag, sich aktiv in die Verhandlungen um den Vertrag einzubringen. Nicht zuletzt die Oppositionsfraktionen waren darum bemüht, der Bundesregierung die Unterstützung des Plenums mitzugeben und dabei auch die Belastbarkeit der BBV zu prüfen. Das Mandat war zwar sehr engmaschig formuliert; in einigen Punkten wurde aber eine abschließende Klärung den Arbeitsgruppen der Regierungskonferenz aufgegeben ${ }^{66}$. Weder die Vertreter der Bundesregierung noch die des Bundestages waren sich deshalb sicher, ob die Beschlüsse von Brüssel die Regierungskonferenz überdauern würden. Dies barg jedoch auch die Gefahr, dass man im Laufe der Konferenz Zugeständnisse hätte machen müssen, die von dem einmal parlamentarisch abgesicherten Mandat nicht erfasst gewesen wären. Die Opposition ging auf Basis der BBV davon aus, dass die Bundesregierung in solchen Fällen erneut Einvernehmen mit dem Bundestag herstellen musste. Entsprechend wurde die Bundesregierung aufgefordert, einen förmlichen Plenumsbeschluss zum Verhandlungsmandat zu erwirken. Die Abgeordneten beriefen sich ausdrücklich auf die erst wenige Monate zuvor von der Bundeskanzlerin eigenhändig unterschriebene BBV, in der es unter VI. „Beitritte und Vertragsrevisionen“ in Abs. 2 S. 1 heißt: „Vor der abschließenden Entscheidung im Rat bemüht sich die Bundesregierung, Einvernehmen mit dem Deutschen Bundestag herzustellen.“

Den Beteiligten war klar, dass die abschließende Entscheidung im Rahmen der Regierungskonferenz fallen würde und nicht erst auf dem Herbstgipfel in Lissabon. Der dortige Beschluss werde reine Formsache sein und der dann vorliegende Text unveränderbar. Nur vor dieser entscheidenden Einigung auf Regierungsebene sei es sinnvoll, ein Einvernehmen

63 So führte der Auswärtige Ausschuss eine ständige Unterrichtung über die Aktivitäten der Bundesregierung im Bereich der GASP ein.

64 Vgl. Website des Rates, http://register.consilium.europa.eu/pdf/de/07/st11/st11218.de07.pdf (Abruf am 27. November 2009).

65 Auch hier sei noch mal an die Grenze des Art. 4 EUV erinnert.

66 Vor allem die Abstimmungsquoren überließ man den Arbeitsgruppen, um den Erfolg des Europäischen Rates nicht zu gefährden. Am Ende kam die Einigung auf Basis der berühmten Ioannina-Formel heraus. 
herzustellen, argumentierten die Vertreter aller Fraktionen ${ }^{67}$. Entscheidend sei, wie im Fall der Grundrechteagentur ${ }^{68}$, die politische Zusage auf Ratsebene, nicht ein Beschluss, der das Ergebnis lediglich noch protokolliere. Auch stelle die Regierungskonferenz einen Teil der Arbeit im Rat dar. Zur Auslegung der BBV wurde zudem herangezogen, dass dieser Abschnitt der Vereinbarung mit „Beitritte“ und „Vertragsrevision“ überschrieben ist, die beiden Entscheidungen somit auf einer Ebene stehen und verfahrensmäßig gleich behandelt werden müssen. So wäre auch bei Beitrittsentscheidungen maßgeblich, ob Verhandlungen aufgenommen werden, und nicht, ob nach 20 Jahren Beitrittsverhandlungen schließlich der Rat den Beitritt (formal) beschließt ${ }^{69}$.

Die Bundesregierung und die Spitzen der Regierungsfraktionen weigerten sich jedoch strikt, den Bundestag über das Mandat abstimmen zu lassen beziehungsweise das Einvernehmen herzustellen. Zwar rechnete man mit größeren Widerständen aus den eigenen Reihen, aber man befürchtete, einen Präzedenzfall in der Anwendung der BBV zu schaffen. Formal beriefen sich Bundeskanzlerin und Außenminister - erneut wie bei der Grundrechteagentur - darauf, dass der förmliche Beschluss über den Vertrag erst fallen werde, wenn die Rechtsexperten die Entscheidungen von Brüssel in einen entsprechenden Text umgewandelt hätten. Dies fiele jedoch bereits in die portugiesische Präsidentschaft. Erst dann wäre es Zeit, dass der Bundestag darüber entscheide. Auch hier wurde also zwischen politischer Einigung und formaler Beschlussfassung im Rat unterschieden, und die politische Einigung sollte, wie bei der Grundrechteagentur, nicht von der Mitwirkung des Parlaments erfasst sein. Insbesondere die Unionsspitzen ${ }^{70}$, die noch am Anfang der Wahlperiode die BBV vor Inkrafttreten der Primärrechtsänderung durchgesetzt hatten, argumentierten plötzlich damit, dass solche Entscheidungen nicht unter die BBV fielen, da diese hierbei nicht im Lichte des Art. 23 Abs. 3 GG, sondern des Abs. 1 auszulegen sei. Bei intergouvernementalen Beschlüssen handele es sich gerade nicht um Rechtsetzungsakte.

Wollte man einen Moment ausmachen, an dem der Bundestag in der 16. Wahlperiode freiwillig, wissentlich und ohne Grund auf Beteiligungsrechte verzichtete, wäre das sicherlich diese von Parlamentariern selbst vorgetragene Argumentation im Juni/Juli 2007. Zum einen stand sie im Gegensatz zu Wortlaut, Teleologie und Historie der BBV, zum anderen widersprach sie dem, was sich die Abgeordneten bei der Schaffung des Art. 23 GG gedacht hatten, denn der Beschlussempfehlung des Maastricht-Ausschusses zur Einführung des Art. 23 GG neue Fassung ist zu entnehmen, dass die Parlamentarier in der 12. Wahlperiode gerade deshalb den Begriff Rechtsetzungsakt gewählt hatten, um sich eine Mitwirkung bei allen Vorhaben der EU, seien sie intergouvernemental oder sekundärrechtlicher Art, vorzubehalten $^{71}$.

67 BT-Plenarprotokoll 16/107, S. 10989C.

68 Auch wenn es sich um verschiedene Verfahren handelt, ist die Diskussion um den Zeitpunkt vergleichbar.

69 Abschnitt VI Ziffer 2 BBV war maßgeblich auf Drängen der CDU im Jahr 2005, vor dem Beschluss, Beitrittsverhandlungen mit der Türkei aufzunehmen, in die BBV aufgenommen worden.

70 Vgl. Volker Kauder, in: BT-Plenarprotokoll 16/107, S. 10989C.

71 Vgl. BT-Drs. 12/3896, S. 24; siehe auch Doris König, Die Übertragung von Hoheitsrechten im Rahmen des europäischen Integrationsprozesses - Anwendungsbereich und Schranken des Artikels 23 des Grundgesetzes, Berlin 2000, S. 391. 
Mit der Entscheidung, nicht auf eine Beteiligung im Vorfeld der Regierungskonferenz zu drängen, verzichtete der Bundestag trotz ausgehandelter Mitwirkung in der BBV auf seine Rechte $^{72}$. Dies sollte weitgehende Folgen haben. Nicht nur der Zeitpunkt des Einvernehmens wurde im weiteren Verlauf der Wahlperiode nach hinten verschoben; auch die Frage, ob es überhaupt eines Einvernehmens bedürfte, wurde von der Bundesregierung immer häufiger gestellt.

Damit hatte der Bundestag zur Hälfte der Wahlperiode ein weiteres wichtiges Kräftemessen mit der Bundesregierung klar verloren. Trotz BBV, die als große Errungenschaft ${ }^{73}$ und wegweisend für andere nationale Parlamente ${ }^{74}$ erklärt wurde, konnten sich die Parlamentarier weder im Falle der Grundrechteagentur noch in der Frage der Mitwirkung bei Primärrechtsänderungen gegenüber der Exekutive durchsetzen. Blieb der klare Wille des Bundestages bei der Grundrechteagentur aufgrund des Verhaltens der Bundesregierung im Rat ungehört, verzichteten die Parlamentarier nur wenige Monate später freiwillig auf die volle Umsetzung der Vereinbarung.

\section{Die Begleitgesetze als Besitzstandswahrung der 15. Wahlperiode}

Die anschließenden Diskussionen um die Begleitgesetze wurden nicht dazu genutzt, diese bis dato erkannten Schwachstellen der Zusammenarbeit zu korrigieren. Sie waren davon geprägt, möglichst wenig gegenüber den institutionellen Anpassungen im Zuge des Verfassungsvertrages zu ändern. Deren Kernstück ist die Einfügung eines ganzen Absatzes in Art. 23 GG. Der neue Abs. 1a enthält zwei Regelungen: Erstens schafft er die innerstaatlichen Voraussetzungen für die Erhebung der Subsidiaritätsklage, zweitens die Grundlage für das Verfahren der Beteiligung der nationalen Parlamente bei vereinfachten Vertragsänderungen ${ }^{75}$. Letzteres wurde ins Grundgesetz aufgenommen, da im Zuge der Beratungen zum Begleitgesetz ${ }^{76}$ im Februar 2008 Zweifel aufkamen, ob die einfachgesetzliche Regelung, wie sie noch das Begleitgesetz zum Verfassungsvertrag $2005^{77}$ vorsah, ausreichen würde ${ }^{78}$.

Nach der parlamentarischen Ratifizierung des Vertrages von Lissabon war dem Europaausschuss letztlich jeder Ehrgeiz, sich für Parlamentsrechte einzusetzen, abhanden gekommen. Wie dargestellt kam es im Vorfeld der Regierungskonferenz zum Vertrag von Lissabon zu Kontroversen über die Auslegung und Ausgestaltung des Einvernehmens gemäß Abschnitt VI Abs. 2 BBV. Konnte sich der Bundestag im Juni/Juli 2007 nicht mit der Ansicht

72 Vor diesem Hintergrund sind die Ausführungen des BVerfG zu Art. 48 Abs. 6 und 7 EUV zu lesen.

73 Vgl. Axel Schäfer / Michael Roth / Christoph Thum, Stärkung der Europatauglichkeit des Bundestages, in: Integration, 16. Jg. (2007), H. 1, S. 45 - 49; Tilmann Hoppe, a.a.O. (Fn. 27).

74 BT-Drs. 16/13169.

75 Deren konkrete Ausgestaltung ist nunmehr im IntVG geregelt.

76 BT-Drs. 16/8489.

77 Gesetz über die Ausweitung und Stärkung der Rechte des Bundestages und des Bundesrates in Angelegenheiten der Europäischen Union, in: BGBl. I 2005, Nr. 71 vom 25. November 2005, S. 3178.

78 Weitere Kritik wäre hier durchaus angebracht. Diese bedürfte jedoch einer intensiven Auseinandersetzung mit der Entscheidung. 
durchsetzen, Einvernehmen stelle zwei übereinstimmende Willenserklärungen dar und habe im Vorfeld der entscheidenden Ratsbeschlüsse (also politische Einigung und nicht Ratssitzungen) zu erfolgen, verzichtete er im Mai 2009 gänzlich auf diesen Anspruch.

Kurz vor Ende der Wahlperiode initiierte die Regierungsfraktion der CDU/CSU einen Antrag, der auf der einen Seite die Erfolge der BBV hervorheben sollte, auf der anderen Seite aber auch Erbpflege hinsichtlich der Bemühungen um die Einhaltung der BBV betreiben wollte. Den neu gewählten Parlamentariern des 17. Bundestags sollten die erkannten Schwachstellen vermittelt und künftige Bundesregierungen an gemachte Zusagen gebunden werden. Dazu wurde im Vorfeld der Einbringung des Antrages ${ }^{79}$ mit dem Auswärtigen Amt verhandelt, inwieweit die Bundesregierung zu Zugeständnissen in der Sache bereit sei. Im Ergebnis erhielten die Vertreter der Regierungsfraktionen die mündliche Zusage der Bundesregierung, sich nochmals mit dem Bundestag über die Frage des Zeitpunktes und der Formalisierung des Einvernehmens zu verständigen. Im daraufhin formulierten Antragstext begrüßte der Bundestag, „dass die Bundesregierung in diesem Schreiben gegenüber dem Deutschen Bundestag auch zusichert, sich bei zukünftigen Unterrichtungen gemäß Ziffer VI BBV bezüglich der Aufnahme von Verhandlungen zur Vorbereitung von Beitritten zur Europäischen Union sowie vor Aufnahme von Verhandlungen zur Änderung der vertraglichen Grundlagen der Europäischen Union an ein vereinbartes formalisiertes Verfahren zur Herstellung des Einvernehmens zwischen Deutschem Bundestag und Bundesregierung zu halten, damit zukünftig Missverständnisse über die Form und den Zeitpunkt der Einvernehmensherstellung ausgeschlossen sind.“

Der Antrag sollte bereits am 28. Mai 2009 abgestimmt werden. Da gemäß $₫ 78$ Abs. 5 GO-BT die Beratungen frühestens am dritten Tag nach Verteilung der Drucksache beginnen ${ }^{80}$ dürfen und der Brief als schriftliche Bestätigung der Bundesregierung am 26. Mai offensichtlich noch nicht bei den Abgeordneten der CDU/CSU angekommen war, wurde der Antrag zunächst nur aufgrund der mündlichen Zusagen als Drucksache verteilt. Das Schreiben erreichte die Abgeordneten am 27. Mai. Darin heißt es jedoch, „dass die Bundesregierung bei künftigen Unterrichtungen über die Aufnahme von Verhandlungen zu Beitritten zur Europäischen Union oder zur Änderung der Vertraglichen Grundlagen der EU im Sinne von Ziffer VI der BBV dies mit folgendem Hinweis verbinden wird: ,Es obliegt jetzt dem Deutschen Bundestag zu entscheiden, zu dieser Unterrichtung nach Ziffer VI der Vereinbarung zwischen dem Deutschen Bundestag und der Bundesregierung über die Zusammenarbeit in Angelegenheiten der Europäischen Union Stellung zu nehmen. “"

Der Brief war eine Provokation. Denn erstens steht dem Bundestag ohnehin zu, bei jedem Vorhaben der Bundesregierung „Stellung zu nehmen“. Und zweitens beschränkte sich dieser Passus lediglich auf „Unterrichtungen im Sinne von Ziffer VI der BBV“. Weder gab es ein Zugeständnis der Bundesregierung, sich künftig an ein formalisiertes Verfahren zu halten, noch eine Zusage, künftig diesen Punkt der BBV im Sinne des Bundestages nachzuverhandeln. Die Bundesregierung stellte nicht nur den Zeitpunkt eines Einvernehmens in Frage, sondern mit der Beschränkung auf „Unterrichtungen“ das Einvernehmen selbst. Auch der Begriff „Stellung nehmen“weist eher auf eine einseitige Handlung des Parlaments

79 BT-Drs. 16/13169.

80 Es besteht in solchen Situationen auch die Möglichkeit, Fristverzicht zu erklären. Hier wurde der Entwurf jedoch am 25. Mai 2009 den Abgeordneten zugeleitet. 
hin als auf eine gemeinsame Einigung. Wie groß die Entrüstung der Parlamentarier war, lässt sich an der Wortwahl selbst von Abgeordneten der Regierungsfraktionen ersehen. ${ }^{81}$

Letztlich spielte sich diese Entrüstung jedoch in der Debatte um den nicht geänderten Antrag zur BBV ab. Konsequent wäre es gewesen, den Antrag an dieser Stelle zu ändern ${ }^{82}$ oder gänzlich zu lassen. So wurde einem Schreiben der Bundesregierung zugestimmt, das in einer späteren Auslegung auch als Nachtragsvereinbarung zur BBV angesehen werden könnte. Der Bundestag verzichtete zum Ende der Wahlperiode nicht nur auf eine erneute Auseinandersetzung mit der Bundesregierung, sondern verteidigte nicht einmal mehr das, was er zu Beginn erreicht hatte.

Die Hilflosigkeit des gesamten Parlaments in der 16. Wahlperiode, im Bereich der Europapolitik und seiner Beteiligungsrechte Tritt zu fassen, lässt das Misstrauen der Verfassungshüter gegenüber dem Gesetzgeber berechtigt erscheinen, denn am Ende zählt eine belastbare Mitwirkungsarchitektur, bei der nicht bei jeder Entscheidung erneut die Teilhabe des Parlaments zur Diskussion gestellt wird. Der Bundestag hat es über viele Jahre nicht vermocht, solche belastbaren Beteiligungsrechte zu erstreiten. Das liegt zum einen daran, dass sich wichtige Führungspersönlichkeiten der Regierungsfraktionen den Regierenden näher fühlen als dem Parlament. Es liegt aber auch daran, dass sich viele Abgeordnete immer noch unzureichend mit europäischen Vorhaben beschäftigen. Außerdem wird die Mitwirkung sicherlich durch die schleichende Informalisierung und Entparlamentarisierung des europäischen Gesetzgebungsprozesses in Mitleidenschaft gezogen ${ }^{83}$. Der Bundestag läuft Gefahr, bloßer Notar der europäischen Gesetzgebung zu werden, und entfernt sich dabei immer mehr von der im GG vorgesehenen Rolle als maßgeblicher Akteur.

$\mathrm{Ob}$ sich an den identifizierten Missständen etwas durch die jetzt neu beschlossenen Beteiligungsrechte ändern wird, die letztlich nur die BBV in Gesetzesform gebracht haben, wird die 17 . Wahlperiode zeigen. Die Zahlen der 16 . Wahlperiode ${ }^{84}$ zeigen zum einen, dass das Argument, mehr parlamentarische Teilhabe führe zur Handlungsunfähigkeit der Exekutive kaum aufrechtzuerhalten ist. Für die zukünftige Regelung der Zusammenarbeit ist ebenfalls eine gesetzliche Ausgestaltung ratsam, denn eine Korrektur der BBV scheiterte vor allem daran, dass der andere Vertragspartner - die Bundesregierung - zustimmen musste, was die Handlungsfähigkeit des Parlaments erheblich einschränkte. Wünschenswert wären künftig auch Befassungspflichten für bestimmte sekundärrechtliche Vorhaben. So könnte - problemlos mit Art. 23 Abs. 3 GG vereinbar - ein genereller Parlamentsvorbehalt in bestimmten Bereichen festgelegt werden, etwa bei finanziellen Zusagen, die den Bundeshaus-

81 So bezeichnete der Abgeordnete Thomas Silberhorn den Brief als „lausiges Schreiben “ und kündigte an, das Schreiben an das Bundesverfassungsgericht, das sich gerade mit den Verfassungsbeschwerden gegen den Vertrag von Lissabon beschäftige, zuzuleiten. BT-Plenarprotokoll 16/224 vom 28. Mai 2009, S. 24697B.

82 Zum Beispiel hätte der entsprechende Passus in den Forderungsteil verschoben werden können.

83 Matthias Ruffert, Entformalisierung und Entparlamentarisierung politischer Entscheidungen als Gefährdung der Verfassung?, in: DVBl 2002, S. 1145.

84 In der Zeit vom 1. September 2007 bis 31. August 2008 wurden dem Bundestag insgesamt 16.400 Dokumente zugeleitet, von denen 1.060 für eine Überweisung in Betracht kamen (insbesondere nach Ziffer 2 der Anlage 8 zur GOBT, also Vorhaben im Sinne der BBV, die grundsätzlich eine Mitwirkung notwendig machen). Zu gerade einmal elf dieser Vorhaben hat der Bundestag eine Stellungnahme abgegeben; vgl. zweiten Monitoringbericht zur Umsetzung der Unterrichtungspflichten der Bundesregierung gemäß der BBV vom 9. Dezember 2008. 
halt belasten. Dies hätte den Vorteil, dass der Bundestag in den politischen Haftungsverband integriert wäre und seine Verantwortung nicht mehr auf „die“ in Brüssel abschieben könnte. Vor allem aber würde die Bundesregierung ihren Verpflichtungen aus Art. 23 Abs. 2 S. 2 GG eigennützig nachkommen, denn das Parlament würde immer erst dann entscheiden, wenn es sich ausreichend informiert fühlt.

Eine der ersten Reaktionen der Vertreter der Bundesregierung am 30. Juni 2009 in Karlsruhe war, dass der „Vertrag“ die Hürde genommen habe und sich die Entscheidung in der Praxis kaum auswirke. Die Aufgabe des Bundestags in der kommenden Wahlperiode wird es sein, dieser Feststellung den Boden zu entziehen und dennoch die Handlungsfähigkeit der Europäischen Union vernünftig zu erhalten.

\title{
Neue (alte) Konfliktlinien in der Europapolitik: Die Parlamentsdebatte zum Vertrag von Lissabon im Deutschen Bundestag
}

\author{
Andreas Wimmel
}

In der Parlamentssitzung vom 24. April 2008 hat der Deutsche Bundestag den Gesetzentwurf zur Ratifikation des EU-Reformvertrages von Lissabon, der die in französischen und niederländischen Referenden gescheiterte „Europäische Verfassung“ ersetzen soll, mit der überwältigenden Mehrheit von 515 Stimmen angenommen. ${ }^{1}$ Die Fraktionen von SPD, FDP und Bündnis 90/Die Grünen sowie eine deutliche Mehrheit der CDU/CSU-Abgeordneten haben, wie allgemein erwartet worden war, dem komplizierten Vertragswerk zugestimmt, während die gesamte Linkspartei sowie sieben CDU/CSU-Vertreter, die ihre Ablehnung in Erklärungen teilweise persönlich begründeten, dagegen votierten. ${ }^{2}$ Damit steht diese Ratifikation in der Tradition einer verlässlichen deutschen Europapolitik: Schon die EU-Verträge von Maastricht (1992) und Nizza (2001) waren von allen Bundestagsfraktionen mit Ausnahme der PDS/Linke Liste mit deutlichen Mehrheiten und ohne große zwischenparteiliche Konflikte ratifiziert worden. ${ }^{3}$ Der breite Konsens der beiden Volkspar-

1 Nach dem erfolgreichen zweiten Referendum in Irland und der Unterzeichnung durch den tschechischen Präsidenten Vaclav Klaus ersetzt seit dem 1. Dezember 2009 der Vertrag von Lissabon die geltenden EU-Grundlagenverträge. Dieser lange Ratifizierungsprozess ändert jedoch prinzipiell nichts an der Positionierung deutscher Parteien zur Europapolitik im Rahmen der Bundestagsdebatte zum Vertrag von Lissabon, die in diesem Beitrag vergleichend dokumentiert wird.

2 Das Endergebnis der namentlichen Abstimmung lautet: 574 abgegebene Stimmen, davon 515 ja, 58 nein, eine Enthaltung. Gegen den Gesetzentwurf stimmten alle 49 Abgeordneten der Partei Die Linke, die sieben CDU/CSU-Abgeordneten Alexander Dobrindt, Herbert Frankenhauser, Peter Gauweiler, Paul Lehrieder, Georg Nüßlein, Marion Seib und Willy Wimmer (Neuss) sowie die beiden fraktionslosen Abgeordneten Henry Nitzsche und Gert Winkelmeier; der Abgeordnete Anton Hofreiter (Bündnis 90/Die Grünen) enthielt sich.

3 Abstimmungsergebnis Nizza: 605 abgegebene Stimmen, davon 571 Ja-Stimmen (alle Abgeordneten von CDU/CSU, SPD und Bündnis 90/Die Grünen), 32 Nein-Stimmen (alle PDS-Abgeord- 